\title{
Corrigendum
}

\section{Corrigendum to 'Molecular simulation of the surface tension of 33 multi-site models for real fluids’ [J. Mol. Liq. 235 (2017) 126-134]}

\section{Stephan Werth, ${ }^{1}$ Simon Stephan, ${ }^{2}$ Martin Thomas Horsch, ${ }^{3}$ and Hans Hasse ${ }^{2}$}

\author{
${ }^{1}$ BorgWarner Turbo Systems GmbH, Marnheimerstr. 85-87, 67292 \\ Kirchheimbolanden, Germany, \\ ${ }^{2}$ Technische Universität Kaiserslautern, Laboratory of Engineering \\ Thermodynamics, Erwin-Schrödinger-Str. 44, 67663 Kaiserslautern, Germany, \\ ${ }^{3}$ UK Research and Innovation, STFC Daresbury Laboratory, Keckwick Ln, \\ Daresbury, Cheshire WA4 4AD, United Kingdom
}

The authors regret that at typesetting stage, additional ' 1 ' digits were mistakenly introduced into the tables, leading to an incorrect reproduction of numerical data. Corrected versions of the tables are included here; the affected values, which in the published article [1] erroneously contained an additional leading digit, are highlighted in bold typeface. The text of the article, and the conclusions formulated therein, were based on the correct data and do not require any modification. The authors would like to apologize for any inconvenience caused.

\section{References}

[1] S. Werth, M. Horsch, H. Hasse, Molecular simulation of the surface tension of 33 multi-site models for real fluids, J. Mol. Liq. 235 (2017) 126-134.

[2] R.P. Danner, R.L. Rowley, W.V. Wilding, J.L. Oscarson, Y. Yang, N.A. Zundel, T.E. Daubert, DIPPR information and data evaluation manager for the design institute for physical properties, AIChE (2013) version 7.0.0.

[3] S. Deublein, P. Metzler, J. Vrabec, H. Hasse, Automated development of force fields for the calculation of thermodynamic properties: Acetonitrile as a case study, Mol. Sim. 39 (2012) 109-118. [4] T. Merker, J. Vrabec, H. Hasse, Molecular simulation study on the solubility of carbon dioxide in mixtures of cyclohexane + cyclohexanone, Fluid Phase Equilib. 315 (2012) 77-83.

[5] T. Merker, J. Vrabec, H. Hasse, Engineering molecular models: Efficient parametrization procedure and cyclohexanol as case study, Soft Mater. 10 (2012) 3-24.

[6] B. Eckl, J. Vrabec, H. Hasse, On the application of force fields for predicting a wide variety of properties: Ethylene oxide as an example, Fluid Phase Equilib. 274 (2008) 16-26.

[7] B. Eckl, J. Vrabec, H. Hasse, Set of molecular models based on quantum mechanical ab initio calculations and thermodynamic data, J. Phys. Chem. B 112 (2008) 12710-12721.

[8] Y.-L. Huang, M. Heilig, H. Hasse, J. Vrabec, Vapor-liquid equilibria of hydrogen chloride, phosgene, benzene, chlorobenzene, ortho-dichlorobenzene, and toluene by molecular simulation, AIChE J. 52 (2011) 1043-1060.

[9] S. Miroshnichenko, T. Grützner, D. Staak, J. Vrabec, Molecular simulation of the vapor-liquid phase behavior of cyanides and their binary mixtures, Fluid Phase Equilib. 354 (2013) 286-297. 
[10] B. Eckl, Y.-L. Huang, J. Vrabec, H. Hasse, Vapor pressure of R227ea + ethanol at $343.13 \mathrm{~K}$ by molecular simulation, Fluid Phase Equilib. 260 (2007) 177-182.

[11] B. Eckl, J. Vrabec, H. Hasse, An optimised molecular model for ammonia, Mol. Phys. 106 (2008) 1039-1046.

[12] T. Schnabel, M. Cortada, J. Vrabec, S. Lago, H. Hasse, Molecular model for formic acid adjusted to vapor-liquid equilibria, Chem. Phys. Lett. 435 (2007) 268-272.

[13] T. Schnabel, A. Srivastava, J. Vrabec, H. Hasse, Hydrogen bonding of methanol in supercritical $\mathrm{CO}_{2}$ : Comparison between ${ }^{1} \mathrm{H}$ NMR spectroscopic data and molecular simulation results, J. Phys. Chem. B 111 (2007) 9871-9878.

[14] T. Schnabel, J. Vrabec, H. Hasse, Molecular simulation study of hydrogen bonding mixtures and new molecular models for mono- and dimethylamine, Fluid Phase Equilib. 263 (2008) 144-159.

[15] Y.-L. Huang, T. Merker, M. Heilig, H. Hasse, J. Vrabec, Molecular modeling and simulation of vapor-liquid equilibria of ethylene oxide, ethylene glycol, and water as well as their binary mixtures, Ind. Eng. Chem. Res. 51 (2012) 7428-7440.

[16] E. Elts, T. Windmann, D. Staak, J. Vrabec, Fluid phase behavior from molecular simulation: Hydrazine, monomethylhydrazine, dimethylhydrazine and binary mixtures containing these compounds, Fluid Phase Equilib. 322-323 (2012) 79-91.

[17] S. Eckelsbach, T. Janzen, A. Köster, S. Miroshnichenko, Y.M. Muñoz Muñoz, J. Vrabec, Molecular models for cyclic alkanes and ethyl acetate as well as surface tension data from molecular simulation, in: M.M. Resch, W.E. Nagel, D.B. Kröner (Eds.), High Performance Computing in Science and Engineering '14, Springer, Heidelberg, 2014, pp. 645-649.

[18] A. Köster, P. Nandi, T. Windmann, D. Ramjugernath, J. Vrabec, Vapor-liquid equilibria of ethylene $\left(\mathrm{C}_{2} \mathrm{H}_{4}\right)+$ decafluorobutane $\left(\mathrm{C}_{4} \mathrm{~F}_{10}\right)$ at 268 to $298 \mathrm{~K}$ from experiment, molecular simulation and the Peng-Robinson equation of state, Fluid Phase Equilib. 336 (2012) 104-112.

[19] M. Thol, F.H. Dubberke, G. Rutkai, T. Windmann, A. Köster, R. Span, J. Vrabec, Fundamental equation of state correlation for hexamethyldisiloxane based on experimental and molecular simulation data, Fluid Phase Equilib. 416 (2016) 133-151.

[20] M. Thol, G. Rutkai, A. Köster, F.H. Dubberke, T. Windmann, R. Span, J. Vrabec, Thermodynamic properties of octamethylcyclotetrasiloxane, J. Chem. Eng. Data 61 (2016) 2580-2595.

DOI of original article: $10.1016 /$ j.molliq.2016.12.062

Corresponding author: Martin Thomas Horsch, STFC Daresbury Laboratory, United Kingdom martin.horsch@stfc.ac.uk 


\section{Table 1}

Molecular models discussed in the present work. The deviations $\delta \rho^{\prime}$ and $\delta p^{s}$ are taken from the referenced publications, and $\delta \gamma$ is the root mean square relative deviation between predictions by the present MD simulations and DIPPR correlations to experimental data [2], determined in the present work. For all considered fluids, the models overestimate $\gamma$ on average. Citations in the "Source" column refer to the literature list of the corrigendum.

\begin{tabular}{|c|c|c|c|c|c|c|c|}
\hline Name & Formula & CAS RN & \# model interaction sites & $\delta \rho^{\prime}$ & $\delta p^{\mathrm{s}}$ & $\delta \gamma$ & Source \\
\hline Acetonitrile & $\mathrm{C}_{2} \mathrm{H}_{3} \mathrm{~N}$ & $75-05-8$ & $3 \mathrm{LJ}+$ dipole & 0.1 & 4.7 & 51.7 & reference [3] \\
\hline Cyclohexane & $\mathrm{C}_{6} \mathrm{H}_{12}$ & $110-82-7$ & $6 \mathrm{LJ}$ & 0.3 & 1.7 & 10.8 & reference [4] \\
\hline Cyclohexanone & $\mathrm{C}_{6} \mathrm{H}_{10} \mathrm{O}$ & $108-94-1$ & 7 LJ + dipole & 0.9 & 2.7 & 26.0 & reference [4] \\
\hline Cyclohexanol & $\mathrm{C}_{6} \mathrm{H}_{10} \mathrm{OH}$ & $108-93-0$ & $7 \mathrm{LJ}+3$ charges & 0.2 & 3.0 & 28.3 & reference [5] \\
\hline Ethylene oxide & $\mathrm{C}_{2} \mathrm{H}_{4} \mathrm{O}$ & $75-21-8$ & $3 \mathrm{LJ}+$ dipole & 0.4 & 1.5 & 16.6 & reference [6] \\
\hline Isobutane & $\mathrm{C}_{4} \mathrm{H}_{10}$ & $75-28-5$ & $4 \mathrm{LJ}+$ dipole + quadrupole & 0.6 & 4.2 & 12.5 & reference [7] \\
\hline Formaldehyde & $\mathrm{CH}_{2} \mathrm{O}$ & $50-00-0$ & $2 \mathrm{LJ}+$ dipole & 0.9 & 4.3 & - & reference [7] \\
\hline Dimethyl ether & $\mathrm{C}_{2} \mathrm{H}_{6} \mathrm{O}$ & $115-10-6$ & $3 \mathrm{LJ}+$ dipole & 0.4 & 2.6 & 18.9 & reference [7] \\
\hline Sulfur dioxide & $\mathrm{SO}_{2}$ & $7446-09-5$ & $3 \mathrm{LJ}+$ dipole + quadrupole & 0.9 & 4.0 & 3.4 & reference [7] \\
\hline Dimethyl sulfide & $\mathrm{C}_{2} \mathrm{H}_{6} \mathrm{~S}$ & $75-18-3$ & $3 \mathrm{LJ}+$ dipole +2 quadrupoles & 0.7 & 4.0 & 18.1 & reference [7] \\
\hline Thiophene & $\mathrm{C}_{4} \mathrm{H}_{4} \mathrm{~S}$ & $110-02-1$ & $5 \mathrm{LJ}+$ dipole + quadrupole & 1.2 & 3.8 & 22.4 & reference [7] \\
\hline Hydrogen cyanide & $\mathrm{HCN}$ & $74-90-8$ & $2 \mathrm{LJ}+$ dipole + quadrupole & 1.0 & 7.2 & 51.9 & reference [7] \\
\hline Nitromethane & $\mathrm{CH}_{3} \mathrm{NO}_{2}$ & $75-52-5$ & $4 \mathrm{LJ}+$ dipole + quadrupole & 0.2 & 18.7 & 31.5 & reference [7] \\
\hline Phosgene & $\mathrm{COCl}_{2}$ & $75-44-5$ & $4 \mathrm{LJ}+$ dipole + quadrupole & 0.5 & 2.1 & 17.2 & reference [8] \\
\hline Benzene & $\mathrm{C}_{6} \mathrm{H}_{6}$ & $71-43-2$ & $6 \mathrm{LJ}+6$ quadrupoles & 0.4 & 3.4 & 11.9 & reference [8] \\
\hline Chlorobenzene & $\mathrm{C}_{6} \mathrm{H}_{5} \mathrm{Cl}$ & $108-90-7$ & $7 \mathrm{LJ}+$ dipole +5 quadrupoles & 0.9 & 5.0 & 17.8 & reference [8] \\
\hline Ortho-dichlorobenzene & $\mathrm{C}_{6} \mathrm{H}_{4} \mathrm{Cl}_{2}$ & $95-50-1$ & $8 \mathrm{LJ}+$ dipole +4 quadrupoles & 0.5 & 6.4 & 34.3 & reference [8] \\
\hline Cyanogen chloride & $\mathrm{NCCl}$ & $506-77-4$ & $3 \mathrm{LJ}+$ dipole + quadrupole & 0.3 & 2.1 & 15.3 & reference [9] \\
\hline Cyanogen & $\mathrm{C}_{2} \mathrm{~N}_{2}$ & $460-19-5$ & $4 \mathrm{LJ}+$ quadrupole & 0.6 & 13.0 & 2.5 & reference [9] \\
\hline Heptafluoropropane (R227ea) & $\mathrm{C}_{3} \mathrm{HF}_{7}$ & $431-89-0$ & 10 LJ + dipole + quadrupole & 1.0 & 1.0 & 7.2 & reference [10] \\
\hline Ammonia & $\mathrm{NH}_{3}$ & $7664-41-7$ & $\mathrm{LJ}+4$ charges & 0.7 & 1.6 & 36.7 & reference [11] \\
\hline Formic acid & $\mathrm{CH}_{2} \mathrm{O}_{2}$ & $64-18-6$ & $3 \mathrm{LJ}+4$ charges & 0.8 & 5.1 & 9.5 & reference [12] \\
\hline Methanol & $\mathrm{CH}_{3} \mathrm{OH}$ & $67-56-1$ & $2 \mathrm{LJ}+3$ charges & 0.6 & 1.1 & 35.3 & reference [13] \\
\hline Dimethylamine & $\mathrm{C}_{2} \mathrm{H}_{7} \mathrm{~N}$ & $124-40-3$ & $3 \mathrm{LJ}+3$ charges & 0.4 & 6.2 & 28.7 & reference [14] \\
\hline Ethylene glycol & $\mathrm{C}_{2} \mathrm{H}_{6} \mathrm{O}_{2}$ & $107-21-1$ & $4 \mathrm{LJ}+6$ charges & 0.8 & 4.8 & 32.6 & reference [15] \\
\hline Water & $\mathrm{H}_{2} \mathrm{O}$ & $7732-18-5$ & $\mathrm{LJ}+3$ charges & 1.1 & 7.2 & 30.7 & reference [15] \\
\hline Hydrazine & $\mathrm{N}_{2} \mathrm{H}_{4}$ & $302-01-2$ & $2 \mathrm{LJ}+6$ charges & 0.5 & 7.6 & 29.2 & reference [16] \\
\hline Methylhydrazine & $\mathrm{CH}_{6} \mathrm{~N}_{2}$ & $60-34-4$ & $3 \mathrm{LJ}+3$ charges & 0.2 & 7.0 & - & reference [16] \\
\hline 1,1-Dimethylhydrazine & $\mathrm{C}_{2} \mathrm{H}_{8} \mathrm{~N}_{2}$ & $57-14-7$ & $4 \mathrm{LJ}+3$ charges & 1.3 & 3.7 & - & reference [16] \\
\hline Ethyl acetate & $\mathrm{C}_{4} \mathrm{H}_{8} \mathrm{O}_{2}$ & $141-78-6$ & $6 \mathrm{LJ}+5$ charges & 0.1 & 4.6 & 10.3 & reference [17] \\
\hline Decafluorobutane & $\mathrm{C}_{4} \mathrm{~F}_{10}$ & $355-25-9$ & $14 \mathrm{LJ}+14$ charges & 0.5 & 3.5 & 10.3 & reference [18] \\
\hline Hexamethyldisiloxane & $\mathrm{C}_{6} \mathrm{H}_{18} \mathrm{OSi}_{2}$ & $107-46-0$ & $9 \mathrm{LJ}+3$ charges & 0.5 & 5.0 & 12.9 & reference [19] \\
\hline Octamethylcyclotetrasiloxane & $\mathrm{C}_{8} \mathrm{H}_{24} \mathrm{O}_{2} \mathrm{Si}_{4}$ & $556-67-2$ & $16 \mathrm{LJ}+8$ charges & 0.5 & 6.0 & 10.5 & reference [20] \\
\hline
\end{tabular}


Table 2

Molecular simulation results for the vapour-liquid equilibrium of the pure components from the present work. The numbers in parentheses indicate the statistical uncertainties in terms of the last given decimal digits.

\begin{tabular}{|c|c|c|c|c|}
\hline $\begin{array}{l}T \\
\mathrm{~K} \\
\end{array}$ & $\begin{array}{l}p^{\mathrm{s}} \\
\mathrm{MPa}\end{array}$ & $\begin{array}{l}\rho^{\prime} \\
\mathrm{mol}^{-1}\end{array}$ & $\begin{array}{l}\rho^{\prime \prime} \\
\mathrm{mol}^{-1}\end{array}$ & $\begin{array}{l}\gamma \\
\mathrm{mN} \mathrm{m}^{-1}\end{array}$ \\
\hline \multicolumn{5}{|c|}{ Acetonitrile } \\
\hline 300 & $0.005(4)$ & 18.885(1) & $0.002(1)$ & $41.9(15)$ \\
\hline 370 & $0.137(38)$ & $17.013(20)$ & $0.053(12)$ & $27.1(6)$ \\
\hline 440 & $0.839(90)$ & $14.810(21)$ & $0.307(58)$ & $15.7(30)$ \\
\hline 510 & $2.72(14)$ & 11.84(9) & $1.09(15)$ & $5.9(3)$ \\
\hline \multicolumn{5}{|c|}{ Cyclohexane } \\
\hline 280 & $0.008(6)$ & $9.355(3)$ & $0.003(1)$ & $29.2(12)$ \\
\hline 335 & $0.049(8)$ & $8.750(4)$ & $0.017(3)$ & $22.3(13)$ \\
\hline 390 & $0.270(33)$ & 8.093(3) & $0.090(9)$ & $15.5(10)$ \\
\hline 445 & $0.855(34)$ & 7.325(13) & $0.273(11)$ & 9.5(7) \\
\hline 510 & 2.387(93) & $6.117(26)$ & $0.834(41)$ & $3.5(4)$ \\
\hline \multicolumn{5}{|c|}{ Cyclohexanone } \\
\hline 250 & $0.000(0)$ & $9.902(8)$ & $0.000(0)$ & $50.6(38)$ \\
\hline 315 & $0.002(2)$ & $9.327(4)$ & $0.001(1)$ & $40.5(12)$ \\
\hline 380 & $0.020(6)$ & 8.741(5) & $0.006(1)$ & $30.8(21)$ \\
\hline 445 & $0.148(22)$ & 8.118(11) & $0.042(5)$ & $22.3(13)$ \\
\hline 510 & $0.558(37)$ & $7.423(14)$ & $0.146(9)$ & $14.8(6)$ \\
\hline \multicolumn{5}{|c|}{ Cyclohexanol } \\
\hline 300 & $0.000(0)$ & $9.700(22)$ & $0.000(0)$ & $40.3(62)$ \\
\hline 375 & $0.009(7)$ & $9.028(4)$ & $0.003(1)$ & $32.0(13)$ \\
\hline 450 & $0.122(20)$ & 8.277(3) & $0.034(8)$ & $22.9(4)$ \\
\hline 525 & $0.633(24)$ & 7.422(13) & $0.165(12)$ & $14.0(7)$ \\
\hline 600 & 2.093(91) & $6.325(20)$ & $0.575(31)$ & $5.7(11)$ \\
\hline \multicolumn{5}{|c|}{ Ethylene oxide } \\
\hline 180 & $0.000(0)$ & $23.230(4)$ & $0.000(0)$ & $48.1(33)$ \\
\hline 245 & $0.006(4)$ & 21.325(6) & $0.004(3)$ & $38.6(23)$ \\
\hline 310 & $0.331(68)$ & $19.323(27)$ & $0.145(30)$ & $25.0(13)$ \\
\hline 375 & $1.304(57)$ & 16.851(13) & $0.495(27)$ & $14.3(19)$ \\
\hline 440 & $4.77(40)$ & $13.39(13)$ & $2.16(34)$ & $3.7(11)$ \\
\hline \multicolumn{5}{|c|}{ Isobutane } \\
\hline 120 & $0.000(0)$ & 12.654(6) & $0.000(0)$ & $37.9(13)$ \\
\hline 185 & $0.001(1)$ & 11.539(3) & $0.001(1)$ & $26.7(8)$ \\
\hline 250 & $0.067(17)$ & $10.383(23)$ & $0.032(7)$ & $17.4(5)$ \\
\hline 315 & $0.584(10)$ & $8.983(28)$ & $0.263(16)$ & 9.1(7) \\
\hline 380 & $2.32(12)$ & $7.112(41)$ & $1.13(12)$ & $2.3(4)$ \\
\hline \multicolumn{5}{|c|}{ Formaldehyde } \\
\hline 180 & $0.001(1)$ & $31.984(54)$ & $0.001(1)$ & $51.6(14)$ \\
\hline 235 & $0.030(9)$ & $29.228(5)$ & $0.017(5)$ & $36.3(15)$ \\
\hline 290 & $0.331(54)$ & 26.222(77) & $0.155(20)$ & $24.2(14)$ \\
\hline 345 & $1.66(13)$ & 22.594(39) & $0.782(66)$ & $12.7(9)$ \\
\hline \multicolumn{5}{|c|}{ Dimethyl ether } \\
\hline 205 & $0.016(8)$ & $17.016(5)$ & $0.016(4)$ & $30.0(12)$ \\
\hline 260 & $0.205(39)$ & $15.415(53)$ & $0.100(35)$ & $19.8(13)$ \\
\hline 315 & $0.95(13)$ & $13.563(13)$ & $0.442(38)$ & $10.9(10)$ \\
\hline 370 & $3.34(35)$ & $10.94(12)$ & $1.70(31)$ & $3.6(6)$ \\
\hline
\end{tabular}


Table 2 (continued)

\begin{tabular}{lllll}
\hline$T$ & $p^{\mathrm{s}}$ & $\rho^{\prime}$ & $\rho^{\prime \prime}$ & $\gamma$ \\
$\mathrm{K}$ & $\mathrm{MPa}$ & $\mathrm{mol} \mathrm{l}^{-1}$ & & $\mathrm{mN} \mathrm{I}^{-1}$ \\
\hline Sulfur dioxide & & & & \\
220 & $0.013(8)$ & $24.771(11)$ & $0.007(4)$ & $39.8(29)$ \\
265 & $0.106(39)$ & $22.923(25)$ & $0.049(14)$ & $29.6(27)$ \\
310 & $0.65(17)$ & $20.932(52)$ & $0.278(74)$ & $20.0(14)$ \\
355 & $1.95(46)$ & $18.48(12)$ & $0.83(19)$ & $11.1(9)$ \\
400 & $4.65(26)$ & $14.87(28)$ & $2.30(25)$ & $3.6(10)$
\end{tabular}

Dimethyl sulfide

190

260

330

400

470

Thiophene

250

320

390

460

530

Hydrogen cyanide

280

315

340

375

410

Nitromethane

260

330

400

470

540

Phosgene

160

225

290

355

420

\section{Benzene}

290

350

410

470

530

Chlorobenzene

250

335

420

505

590
0.001(1)

$0.036(14)$

$0.342(26)$

1.70(15)

5.32(33)

$0.002(1)$

$0.035(16)$

$0.263(37)$

1.12(11)

3.13(29)

$0.038(21)$

$0.122(12)$

$0.343(32)$

$0.949(50)$

2.03(10)

$0.001(1)$

$0.030(7)$

$0.109(22)$

$0.826(69)$

2.84(26)

$0.000(0)$

0.007(0)

$0.133(34)$

$0.937(36)$

3.35(33)

$0.007(7)$

0.092(23)

$0.423(47)$

1.441(67)

3.337(92)

$0.004(3)$

$0.011(6)$

$0.122(13)$

$0.79(14)$

2.91(8)
19.996(5)

18.300(17)

16.471(18)

14.315(56)

10.85(26)

13.107(7)

12.168(6)

11.166(7)

10.001(16)

8.458(65)

25.756(38)

24.015(12)

22.589(23)

20.50(12)

17.766(69)

19.569(16)

18.014(6)

16.259(52)

14.337(25)

11.70(20)

17.047(11)

15.578(10)

14.047(7)

12.311(20)

9.873(48)

11.208(13)

10.403(7)

9.523(3)

8.450(19)

6.884(36)

10.320(8)

9.466(11)

8.567(23)

7.520(30)

5.992(20)
$0.000(0)$

$0.022(14)$

$0.134(16)$

$0.635(65)$

2.60(57)

$0.001(1)$

$0.013(4)$

$0.084(9)$

$0.342(35)$

1.01(16)

$0.021(11)$

$0.063(10)$

$0.153(23)$

0.431(41)

1.00(12)

48.6(16)

36.1(10)

23.6(7)

11.8(13)

2.1(8)

43.6(22)

33.3(7)

22.4(13)

13.6(10)

5.0(8)

34.0(11)

25.5(21)

19.2(12)

13.2(17)

7.6(7)

55.3(37)

0.001(1)

0.014(6)

$0.044(15)$

$0.266(32)$

1.06(15)

41.6(34)

28.3(14)

16.5(15)

6.0(9)

49.2(32)

34.8(17)

23.1(21)

12.5(12)

3.7(7)

33.2(13)

24.3(7)

15.7(10)

8.5(11)

2.6(9)

1.21(13)

46.7(28)

33.3(19)

21.9(8)

11.7(9)

3.2(9) 
Table 2 (continued)

\begin{tabular}{|c|c|c|c|c|}
\hline $\begin{array}{l}T \\
\mathrm{~K}\end{array}$ & $\begin{array}{l}p^{\mathrm{s}} \\
\mathrm{MPa}\end{array}$ & $\begin{array}{l}\rho^{\prime} \\
\mathrm{mol} \mathrm{l}^{-1}\end{array}$ & $\begin{array}{l}\rho^{\prime \prime} \\
\mathrm{mol} \mathrm{l}^{-1}\end{array}$ & $\begin{array}{l}Y \\
\mathrm{mN} \mathrm{m}^{-1}\end{array}$ \\
\hline \multicolumn{5}{|c|}{ Ortho-dichlorobezene } \\
\hline 375 & $0.003(2)$ & 8.472(5) & $0.001(1)$ & $36.1(51)$ \\
\hline 470 & $0.097(17)$ & 7.631(3) & $0.026(6)$ & $23.4(8)$ \\
\hline 565 & $0.701(89)$ & $6.647(16)$ & $0.173(28)$ & $12.6(15)$ \\
\hline 660 & $2.79(14)$ & $5.240(33)$ & $0.807(80)$ & $3.0(7)$ \\
\hline \multicolumn{5}{|c|}{ Cyanogen chloride } \\
\hline 280 & $0.079(21)$ & 19.777(13) & $0.036(8)$ & $28.3(21)$ \\
\hline 315 & $0.312(36)$ & $18.490(46)$ & $0.135(9)$ & 21.6(16) \\
\hline 350 & $0.86(17)$ & $17.08(39)$ & $0.361(83)$ & $14.9(21)$ \\
\hline 385 & $1.86(43)$ & $15.27(7)$ & $0.79(23)$ & 9.4(17) \\
\hline 420 & $3.74(36)$ & 12.92(9) & $1.86(54)$ & $4.2(7)$ \\
\hline \multicolumn{5}{|c|}{ Cyanogen } \\
\hline 260 & $0.094(31)$ & 18.030(33) & $0.049(21)$ & $21.9(24)$ \\
\hline 290 & $0.349(95)$ & $16.866(58)$ & $0.168(25)$ & $16.6(12)$ \\
\hline 320 & $0.79(30)$ & 15.546(99) & $0.36(17)$ & $11.5(16)$ \\
\hline 350 & $2.17(34)$ & 13.820(16) & $1.04(26)$ & $5.7(6)$ \\
\hline 380 & $4.40(5)$ & $11.15(28)$ & $2.89(40)$ & 1.2(6) \\
\hline \multicolumn{5}{|c|}{ Heptafluoropropane } \\
\hline 200 & $0.002(1)$ & $10.364(13)$ & $0.001(1)$ & $21.9(10)$ \\
\hline 250 & $0.063(10)$ & $9.381(8)$ & $0.031(2)$ & 14.8(19) \\
\hline 300 & $0.466(39)$ & 8.215(3) & $0.213(21)$ & 7.7(9) \\
\hline 350 & $1.776(68)$ & $6.531(20)$ & $0.938(46)$ & $1.8(6)$ \\
\hline \multicolumn{5}{|c|}{ Ammonia } \\
\hline 220 & $0.029(2)$ & 41.985(19) & $0.015(1)$ & 55.1(19) \\
\hline 260 & $0.233(38)$ & $38.927(14)$ & $0.116(23)$ & $40.1(36)$ \\
\hline 300 & $1.029(80)$ & $35.470(30)$ & $0.467(39)$ & 26.2(13) \\
\hline 340 & $3.080(66)$ & $31.410(26)$ & $1.421(43)$ & $15.2(25)$ \\
\hline 380 & $7.23(15)$ & $25.572(21)$ & $3.86(21)$ & $4.8(1)$ \\
\hline \multicolumn{5}{|c|}{ Formic acid } \\
\hline 300 & $0.014(10)$ & 26.192(5) & $0.007(2)$ & 39.3(29) \\
\hline 360 & $0.072(9)$ & 24.478(9) & $0.038(14)$ & $30.2(23)$ \\
\hline 420 & $0.333(28)$ & 22.607(11) & $0.169(20)$ & $22.0(15)$ \\
\hline 480 & $1.14(17)$ & $20.36(17)$ & $0.543(61)$ & 15.3(13) \\
\hline 540 & $3.20(25)$ & $17.45(14)$ & $1.57(18)$ & 6.7(15) \\
\hline \multicolumn{5}{|c|}{ Methanol } \\
\hline 245 & $0.001(1)$ & 26.175(9) & $0.001(1)$ & $36.0(16)$ \\
\hline 320 & $0.045(17)$ & 23.958(6) & $0.020(7)$ & 27.1(16) \\
\hline 395 & $0.58(13)$ & 21.317(36) & $0.359(49)$ & $14.0(20)$ \\
\hline 470 & $3.61(14)$ & 17.318(91) & 1.61(26) & $6.0(14)$ \\
\hline \multicolumn{5}{|c|}{ Dimethylamine } \\
\hline 210 & $0.003(1)$ & 16.653(10) & $0.002(1)$ & $35.2(13)$ \\
\hline 260 & $0.033(11)$ & $15.523(4)$ & $0.016(6)$ & 27.3(4) \\
\hline 310 & $0.257(24)$ & $14.279(7)$ & $0.109(10)$ & $19.0(2)$ \\
\hline 360 & $1.04(10)$ & $12.888(6)$ & $0.407(45)$ & 11.8(9) \\
\hline 410 & $2.65(74)$ & $10.814(77)$ & $1.07(50)$ & $5.3(18)$ \\
\hline
\end{tabular}


Table 2 (continued)

\begin{tabular}{|c|c|c|c|c|}
\hline $\begin{array}{l}T \\
\mathrm{~K}\end{array}$ & $\begin{array}{l}p^{\mathrm{s}} \\
\mathrm{MPa}\end{array}$ & $\begin{array}{l}\rho^{\prime} \\
\mathrm{mol} \mathrm{l}^{-1}\end{array}$ & $\begin{array}{l}\rho^{\prime \prime} \\
\mathrm{mol} \mathrm{l}^{-1}\end{array}$ & $\begin{array}{l}Y \\
\mathrm{mN} \mathrm{m}^{-1}\end{array}$ \\
\hline \multicolumn{5}{|c|}{ Ethylene glycol } \\
\hline 380 & $0.005(3)$ & 16.867(29) & $0.002(1)$ & $65.6(21)$ \\
\hline 480 & $0.107(60)$ & $15.527(25)$ & $0.028(11)$ & $42.3(36)$ \\
\hline 580 & $1.144(52)$ & 13.759(14) & $0.268(9)$ & 23.6(31) \\
\hline 680 & $5.45(58)$ & 10.906(97) & $1.44(19)$ & $6.2(12)$ \\
\hline \multicolumn{5}{|c|}{ Water } \\
\hline 300 & $0.006(5)$ & $56.348(12)$ & $0.002(1)$ & $94.0(21)$ \\
\hline 375 & $0.069(16)$ & $52.650(12)$ & $0.024(8)$ & 75.1(13) \\
\hline 450 & $0.63(10)$ & $48.475(11)$ & $0.31(13)$ & 56.9(19) \\
\hline 525 & 3.31(16) & 43.412(11) & $0.94(6)$ & $34.1(21)$ \\
\hline 600 & $10.43(20)$ & $36.485(46)$ & $3.28(3)$ & 13.7(20) \\
\hline \multicolumn{5}{|c|}{ Hydrazine } \\
\hline 280 & $0.000(0)$ & 32.321(8) & $0.000(0)$ & $96.7(23)$ \\
\hline 360 & $0.028(10)$ & $29.955(5)$ & $0.010(5)$ & $72.6(13)$ \\
\hline 440 & $0.358(24)$ & $27.220(18)$ & $0.153(19)$ & 49.4(30) \\
\hline 520 & $2.24(64)$ & 24.063(80) & 1.16(33) & 29.9(20) \\
\hline \multicolumn{5}{|c|}{ Methylhydrazine } \\
\hline 270 & $0.009(5)$ & 19.384(10) & $0.004(3)$ & $57.3(13)$ \\
\hline 345 & $0.031(13)$ & $17.944(6)$ & $0.012(3)$ & 43.1(16) \\
\hline 420 & $0.420(64)$ & $16.358(48)$ & $0.137(40)$ & $29.7(12)$ \\
\hline 495 & $1.93(21)$ & $14.598(16)$ & $0.573(51)$ & 16.7(8) \\
\hline 570 & $5.21(22)$ & $12.21(12)$ & $1.73(13)$ & $5.7(9)$ \\
\hline \multicolumn{5}{|c|}{ 1,1-Dimethylhydrazine } \\
\hline 260 & $0.003(2)$ & $13.783(6)$ & $0.001(1)$ & $35.5(21)$ \\
\hline 315 & $0.045(13)$ & $12.833(4)$ & $0.018(6)$ & 27.6(16) \\
\hline 370 & $0.290(14)$ & $11.806(6)$ & $0.101(9)$ & 19.4(9) \\
\hline 425 & $1.03(13)$ & 10.603(3) & $0.344(40)$ & $11.9(4)$ \\
\hline 480 & 2.97(19) & $9.026(33)$ & $1.10(12)$ & 4.6(4) \\
\hline \multicolumn{5}{|c|}{ Ethylacetate } \\
\hline 190 & $0.000(0)$ & $11.726(4)$ & $0.000(0)$ & 42.4(19) \\
\hline 265 & $0.002(1)$ & $10.709(18)$ & $0.001(1)$ & $29.9(6)$ \\
\hline 340 & $0.063(12)$ & $9.636(10)$ & $0.024(3)$ & 19.7(9) \\
\hline 415 & $0.497(39)$ & $8.379(6)$ & $0.170(6)$ & $10.9(12)$ \\
\hline 490 & $2.078(53)$ & 6.584(19) & $0.831(37)$ & $3.0(7)$ \\
\hline \multicolumn{5}{|c|}{ Decafluorobutane } \\
\hline 260 & $0.077(13)$ & $6.848(12)$ & $0.038(7)$ & $11.8(17)$ \\
\hline 310 & $0.357(6)$ & $6.021(28)$ & $0.157(5)$ & 6.5(6) \\
\hline 360 & $1.329(67)$ & 4.852(77) & $0.657(43)$ & $1.7(3)$ \\
\hline \multicolumn{5}{|c|}{ Hexamethyldisiloxane } \\
\hline 210 & $0.000(0)$ & $5.211(31)$ & $0.000(0)$ & 26.3(17) \\
\hline 280 & $0.002(1)$ & $4.788(5)$ & $0.001(0)$ & $18.4(9)$ \\
\hline 350 & $0.047(15)$ & 4.327(8) & $0.017(4)$ & 12.1(11) \\
\hline 420 & $0.299(20)$ & 3.771(10) & $0.097(10)$ & $6.7(4)$ \\
\hline 490 & $1.161(26)$ & $3.020(25)$ & $0.421(10)$ & $1.8(2)$ \\
\hline
\end{tabular}


Table 2 (continued)

\begin{tabular}{lllll}
\hline$T$ & $p^{\mathrm{s}}$ & $\rho^{\prime}$ & $\rho^{\prime \prime}$ & $\gamma$ \\
$\mathrm{K}$ & $\mathrm{MPa}$ & $\mathrm{mol} \mathrm{l}^{-1}$ & $\mathrm{~mol} \mathrm{l}^{-1}$ & $\mathrm{mN} \mathrm{m}^{-1}$ \\
\hline \multicolumn{2}{l}{ Octamethylcyclotetrasiloxane } & & & \\
310 & $0.000(0)$ & $\mathbf{3 . 1 3 5 ( 1 4 )}$ & $0.000(0)$ & $18.6(33)$ \\
355 & $0.004(2)$ & $\mathbf{2 . 9 7 0 ( 1 2 )}$ & $0.001(0)$ & $14.6(10)$ \\
420 & $0.044(14)$ & $\mathbf{2 . 7 0 9 ( 8 )}$ & $0.013(4)$ & $10.1(11)$ \\
485 & $0.217(15)$ & $\mathbf{2 . 4 0 3 ( 1 2 )}$ & $0.061(4)$ & $\mathbf{5 . 7 ( 2 )}$ \\
550 & $0.699(12)$ & $\mathbf{1 . 9 8 5 ( 2 7 )}$ & $0.211(7)$ & $\mathbf{1 . 9 ( 3 )}$
\end{tabular}

